\title{
Classification of Full-Thickness Traumatic Macular Holes by Optical Coherence Tomography
}

\author{
Jingjing Huang, $\mathrm{MD}^{1}$, Xing Liu, MD, $\mathrm{PhD}^{1}$, Ziqiang $\mathrm{Wu}, \mathrm{MD}^{2}$, Xiaofeng Lin, MD, Ph.D ${ }^{1}$, Mei \\ $\mathbf{L i}, \mathbf{M D}{ }^{1}$, Laurie Dustin, $\mathbf{M S}^{3}$, and Srinivas Sadda, MD ${ }^{4}$ \\ ${ }^{1}$ State Key Laboratory of Ophthalmology, Zhongshan Ophthalmic Center, Sun Yat-sen University, \\ Guangzhou, China \\ ${ }^{2}$ Center for Advanced Eye Care, Carson City, NV \\ ${ }^{3}$ Department of Preventive Medicine, Keck School of Medicine at University of Southern \\ California, Los Angeles, CA \\ ${ }^{4}$ Doheny Image Reading Center, Doheny Eye Institute, Los Angeles, CA
}

\begin{abstract}
Purpose-To describe morphological features of traumatic macular holes (TMH) on optical coherence tomography (OCT) and to correlate them with clinical findings.

Methods-Seventy-three consecutive patients diagnosed with full-thickness TMH involving the fovea underwent complete ophthalmic evaluation followed by horizontal and vertical line scans using the StratusOCT. Retinal thickness at the edges of the holes, apical and basal diameters, and various clinical parameters were recorded. The approximate apical and basal areas and eccentricities of the holes were calculated. Morphological parameters were correlated with clinical findings.
\end{abstract}

Results-Based on OCT findings, TMHs were classified into 5 morphological types with varying average retinal thicknesses, apical areas, and basal areas. Patients who presented more than 90 days after injury had greater average retinal thickness $(\mathrm{p}=0.03)$ and apex areas $(\mathrm{p}=0.002)$ compared to those who presented within 90 days. Older patients developed more circular holes, i.e. less eccentricity of the apex $(\mathrm{p}=0.04)$ and base $(\mathrm{p}=0.01)$. Interestingly, none of the morphological parameters investigated in the current study correlated with visual acuity. Patients who presented later in the clinical course or who had greater average retinal thicknesses tended to have better vision ( $\mathrm{p}=0.11$ and $\mathrm{p}=0.07$, respectively).

Conclusions-OCT evaluation may yield important insights into the pathogenesis and clinical findings of traumatic macular holes.

\section{Keywords}

optical coherence tomography; traumatic macular hole

Correspondence to Xing Liu, MD, PhD. State Key Laboratory of Ophthalmology, Zhongshan Ophthalmic Center, Sun Yat-sen University, 54 Xianlienan Road, Guangzhou 510060, China. Telephone: 8620-87330434, Fax: 8620-87333271, E-mail: drliuxing@163.com.

Conflict of Interest: The authors have no proprietary interest in any of the topics discussed in this manuscript. 
Optical coherence tomography (OCT), which can provide detailed cross-sectional images of the retina, has refined Gass's original classification and description of idiopathic macular holes (IMHs). 1,2 It is now thought that both anteroposterior and tangential vitreous traction are involved in the pathogenesis of IMH. Traumatic macular holes (TMHs), on the other hand, are usually associated with forceful blunt injury, although they have also been reported after inadvertent Neodymium:YAG laser.3 First described in 1869, TMH was originally hypothesized to be caused by direct impact on the eyeball.4,5 However, it is now apparent that TMH may occur instantly after injury or manifest days or weeks later. Immediate vision loss is probably due to foveal detachment, while progression of cystic changes and photoreceptor loss may account for the delayed visual compromise in some patients.6,7 In one series, TMHs were found in only $1.4 \%$ of closed eye trauma patients and in $0.15 \%$ of open eye trauma patients. 8 Due to the low incidence of TMH, relatively few papers have studied its clinical and OCT characteristics.9 Because of the variability in the force of impact and in the material characteristics of the inflicting object, the characteristics of the resultant TMHs may be different. The objective of our study is to identify various morphologic types of TMHs using OCT imaging, and to correlate them with other clinical findings.

\section{Methods}

Charts of 73 consecutive patients diagnosed with unilateral TMH at the outpatient clinics of Zhongshan Ophthalmic Center of Sun Yat-sen University (Guangzhou, China) from January 2005 to June 2006 were retrospectively reviewed. The study was conducted in accordance with the tenets of the Declaration of Helsinki and was approved by the Institutional Review Board. Informed consent was obtained from all patients.

The inclusion criteria were: 1) reliable history of eye trauma and no prior intraocular surgery; 2) self-reported significant decrease of visual acuity within 4 weeks of trauma; 3 ) full-thickness macular hole involving the foveal center, demonstrated on dilated stereoscopic examination and on OCT; 4) no retinal detachment except for that related to the macular hole; 5) no history of high myopia (no more than 6D); 6) no significant ocular pathology other than macular hole at the time of evaluation.

All subjects underwent complete ophthalmic evaluation including best-refracted Snellen visual acuity, slit-lamp biomicroscopy, dilated stereoscopic examination, and fundus photography. The presence or absence of posterior vitreous detachment (PVD), cuff of subretinal fluid under the TMH, and general shape of the hole were noted during each clinical examination. For each patient, a number of clinical characteristics were recorded including gender, age at the time of injury, the time between injury and examination, and if known, the precise mechanism of trauma (e.g. type of inflicting object). The maculae were imaged using the StratusOCT (Carl Zeiss, Dublin, CA, USA) line scan protocol, with resolution of $512 \mathrm{~A}$-scans over a scan length of $5 \mathrm{~mm}$. The horizontal and vertical scans for each eye were performed by a trained physician $(\mathrm{JJH})$, with attempts to ensure centration on the hole. 
Quantitative measurements (in $\mu \mathrm{m}$ ) using the StratusOCT software's calipers were performed according to the following definitions: retinal thickness was the distance from the outer border of the neurosensory retina to the internal limiting membrane (i.e. areas of subretinal fluid were not included in the retinal thickness measurement); the apical diameter of macular hole was the length of the narrowest neurosensory retinal defect; and the basal diameter of macular hole was the widest distance at the base of the hole, immediately above the retinal pigment epithelium. The nasal, temporal, superior, and inferior thicknesses of the neurosensory retina at the apical margin of the hole were measured. The average retinal thickness was the mean of these four measurements. Assuming all TMHs to be approximately elliptical, we defined $a$ to be the radius of the larger axis (the longer of the two apical radii obtained by the vertical and horizontal line scans) and $b$ as the radius of the smaller axis of the ellipse. The apical area was thus $\pi a b$, in $\mu \mathrm{m}^{2}$. The apical eccentricity was calculated by the formula:

$$
\varepsilon=\sqrt{1-\frac{b^{2}}{a^{2}}}
$$

The basal area and eccentricity were similarly computed. Eccentricity is a measure of the circularity of an ellipse. When the major and minor axes are equal in length, i.e. a circle, the eccentricity is zero. However, if the major axis is much longer than the minor axis, the eccentricity approaches 1 .

Because of the unknown reliability of time from injury to exam among patients who presented late in the clinical course, subgroup analyses were performed among the 58 patients who presented within 90 days of injury.

\section{Statistical Methods}

SAS version 9.1 (SAS Institute, Cary, NC, USA) programming language was used for all analyses. Analysis of variance (ANOVA) was used to compare means by types of macular hole. Multiple pairwise comparisons were performed when ANOVA $\mathrm{p}$ was $<0.05$, adjusting for the number of pairs tested using a Bonferroni correction. Categorical variables were compared using Chi-square tests.

\section{Results}

The average age of the 73 patients was $27.1 \pm 9.2$ years, with $89.0 \%$ below age 40 (range 8 to 55 years). There were 63 males and 10 females. The right eye was affected in 36 patients, while 37 sustained left eye injury. The substances which caused the trauma included but were not limited to balls, fists, flares, and stones.

The best corrected visual acuity ranged from hand motion to 20/40 (median 20/200). The average $\log$ Mar visual acuity, defined as - $\log _{10}$ (visual acuity expressed as decimal), was $1.23 \pm 0.51$ at the time of initial evaluation, or equivalent to approximately 20/340 vision. The average time elapsed between injury and presentation was $151 \pm 550$ days (range 2 days to 12 years, median 29 days). Among the 58 patients who presented within 90 days, the average 
$\log$ Mar visual acuity was $1.25 \pm 0.53$ (or 20/356 vision), and the average time elapsed between injury and presentation was $24.2 \pm 20.9$ days (median 15 days).

Per the study criteria, all of the TMHs were full-thickness defects involving the fovea at the time of presentation. No vitreous detachment was noted in any of the patients either on examination or evident on OCT. Using their OCT morphology, the 73 cases of TMH were classified into 5 types by an experienced OCT reader (LX):

- $\quad$ Type I - macular holes with cystic edema of the neurosensory retina on both margins of the hole (Fig. 1) on both the horizontal and vertical scans. Morphologically these TMHs were similar to idiopathic macular holes -- 20 cases $(27.4 \%)$.

- $\quad$ Type II - macular holes with cystic edema of the neurosensory retina on only one margin of the hole (Fig. 2) on either the horizontal or vertical scan - 18 cases $(24.7 \%)$.

- $\quad$ Type III - macular hole with full-thickness defect of neurosensory retina without cystic edema or detachment of the margins (Fig. 3) -- 14 cases (19.2\%).

- $\quad$ Type IV - macular hole with localized detachment of the neurosensory retina at the margin without cystic edema (Fig. 4) -- 17 cases (23.3\%).

- $\quad$ Type V - macular hole with thinning of the neurosensory retina (Fig. 5) -- 4 cases $(5.5 \%)$.

The characteristics of the 73 patients are summarized on Table 1a. Pairwise comparisons among the groups reveal that: 1) As expected, patients with Type I and Type II TMHs, which by definition had macular edema, had greater average retinal thickness than the others; 2 ) Type IV TMHs, owing to the presence of localized retinal detachment, had significantly greater basal areas compared to the other types, but Type V TMHs had greater apical areas than Type IV holes; 3) Patients with Type I TMHs presented later than patients with Type IV; 4) There were no significant differences in terms of gender, age, visual acuity and eccentricity (apical and basal) among the different types of TMHs.

The clinical characteristics of the subgroup of 58 patients who presented within 90 days are summarized on Table 1b. Pairwise comparisons revealed similar findings. Additionally, Type III TMH patients were older than those with Type I, and Type V TMHs had greater apical areas than all other types. Among patients who presented more than 90 days after injury, Type I TMHs were over-represented ( 7 of 15 patients, or $46.7 \%$, Table 2 ), even though Type I TMHs were only $27.4 \%$ of the 73 total cases in the study.

The areas and eccentricities of TMHs, whether apical or basal, did not correlate with visual acuity (Table 3). This was true among all TMH types. Patients who presented later in the clinical course or who had greater average retinal thicknesses tended to have better vision $(\mathrm{p}=0.13$ and $\mathrm{p}=0.07$, respectively).

Patients who presented more than 90 days after injury had greater average retinal thicknesses $(\mathrm{p}=0.03)$ and larger apical areas $(\mathrm{p}=0.002)$ than those who were evaluated within 90 days (Table 4). Similarly there was significant correlation between average retinal thickness and 
the duration of days from injury to evaluation ( $\mathrm{r}=0.347, \mathrm{p}=0.003$ ) (Figure 6). Interestingly, age of the patient was inversely correlated with apical $(\mathrm{p}=0.04)$ and basal $(\mathrm{p}=0.01)$ eccentricities (Table 5), but not with the apical and basal areas, visual acuity, and average retinal thickness.

\section{Discussion}

This study evaluated the OCT morphometric characteristics of 73 consecutive patients with traumatic macular holes and correlated them with other clinical variables in order to gain further insights into the pathogenesis of these lesions. We also categorized full-thickness TMHs into five major morphometric types.

\section{Mechanisms of traumatic macular hole formation}

Yamashita et al thought that there were 2 types of TMHs.10 One type forms immediately after trauma, with avulsion and rupture of fovea causing acute visual loss. The other type occurred more gradually, from several days to months after trauma. The mechanism was similar to that of vitreomacular traction syndrome. Histological findings confirmed that some cases of TMHs did not occur immediately after trauma, but through a development of macular edema and cysts.11 Although the current investigation does not seek to discern the onset of macular hole, it is worthwhile to consider the implications of these two possible mechanisms in relation to the findings of the present study.

- Rapid onset traumatic macular hole-Early study with high-speed photography found that blunt trauma resulted in sudden anteroposterior compression and equatorial expansion of the globe that produced significant stress on the retina at points of vitreous attachment.12 Because young patients, the group most epidemiologically associated with blunt trauma, often have the greatest vitreofoveal adherence, the sudden traction on the anatomically thin fovea can more easily result in a macular hole. This mechanism may be responsible for those cases in which the TMH occurs immediately after injury. The finding that the incidence of TMH was higher in closed eye trauma than in open eye trauma also supports this proposed mechanism. 8 Open eye trauma presumably would cause less equatorial expansion as the ocular content may exude out of the wound rather than be compressed laterally. Additionally, objects that cause open eye trauma are likely sharper, thus exerting less anteroposterior force.

If this proposed mechanism is true, then patients with vitreous detachment at the fovea at the moment of injury would presumably have a lower risk of developing TMH. We found no PVD in any of the 73 eyes, documented either on careful clinical examination or on StratusOCT. In a study of 20 patients with TMHs, posterior vitreous detachment (PVD) was found in only three eyes (15\%), and the vitreous was detached from the macula in only one (5\%).6 Among another 25 patients with TMHs, 84\% had attached posterior vitreous at the time of vitrectomy. 13 Both of these studies had a statistically significant different PVD rate than ours (Fisher exact test $p=0.01$ and $p=0.006$, respectively). The difference may relate to different methods of detecting PVD or demographic differences of the patient populations. Additionally, PVD seen at the time of evaluation in some of these case reports could have been a result of the injury, rather than an antecedent event. 
A similar mechanism that does not necessarily require vitreous traction would be if the equatorial expansion directly stretches the posterior pole, resulting in the fovea being pulled apart. Using indocyanine green staining during vitrectomy, ILM rupture could be detected in some traumatized eyes.14 It was hypothesized that macular holes occur because the elasticity and strength of Bruch's membrane and of the ILM are not as great as those of the sclera. 14

Because the sudden tractional force caused by blunt trauma is not as even as that causing IMH, a variety of TMH morphologies may arise. Whereas IMHs are usually round, TMHs are thought to be elliptical or have irregular edges like retinal tears, 6 i.e. greater eccentricity. However, a recent OCT study of TMHs revealed that $91.7 \%$ were round at the time of presentation.9 The discrepancy may be due to a much longer time from injury to diagnosis (average 8.1 months, or approximately 245 days) in that study. It is therefore possible that TMHs start out elliptical or irregular but gradually become more circular. This phenomenon has been documented by several other case reports.7,15,16

- Delayed-onset traumatic macular hole-In a case report, there was no detectable macular hole immediately after injury, but foveal detachment was found the next day.15 A full thickness macular hole with asymmetric cystic edema of the neurosensory retina at the margin (Type II) could be discerned 4 weeks later. A similar case was described in which no TMH could be discerned by OCT until two weeks after trauma.17 Tornambe believed that disruption in the internal retinal layers with secondary vitreous fluid accumulation can cause intraretinal swelling and macular hole formation.18 ILM rupture may potentiate the accumulation of fluid in the inner retina.14,19

Persistent vitreofoveal adhesion may lead to delayed TMH formation.10 That vitrectomy appeared to be effective in resolving delayed-onset TMHs supports the hypothesis that the vitreous plays an important role in the formation of this type of TMHs also.20,21

Based on histological findings, Coats believed that TMH develop secondary to cystic macular edema.11 However, some cases with detailed follow up did not demonstrate cysts prior to TMH formation.10,15 Cystic macular edema may result in lamellar holes instead of full-thickness holes. Patients who undergo retinal cystic changes after injury may develop what appears to be foveal atrophy, and are thus missed by investigations focusing on full thickness holes. Since the presence of a full-thickness macular hole was an inclusion criteria, our study did not address this question. Further research may elucidate whether retinal cyst formation is an important precursor in the pathogenesis of some TMHs.

We found age to be inversely related to apical and basal eccentricity, suggesting that older patients had more circular TMHs. Because older patients tend to have weaker vitreofoveal adhesion, injury may be less likely to result in a rapid-onset TMH but instead may lead to a more gradual sequence of events prior to TMH formation. Whereas vitreofoveal avulsion forces are sudden and would likely lead to irregular edges, a prolonged process would presumably allow a more circular hole to develop as forces have more time to distribute evenly around the fovea. 


\section{Classification and evolution of TMHs documented by OCT}

OCT shows the cross-sectional morphology of macular holes, maps the vitreoretinal interface, and makes quantitative measurements possible. 22 The importance of OCT in the identification and characterization of idiopathic macular holes has been amply demonstrated. 23,24 However, few investigators have employed it in studying traumatic macular holes.

Two TMH cases reported by Yamada et al showed that both Type II and Type III TMHs may evolve into Type I.15 In the present study, among the cases evaluated more than 90 days after the initial trauma, Type I TMHs predominated. Patients who presented later also had significantly greater retinal thickness ( $\mathrm{p}=0.03$, Table 4 ), which was highest among Type I patients. These findings suggest that some TMHs may evolve into Type I over time, with increase in retinal edema (Figure 6). Possible mechanisms include injury-induced abnormal capillary permeability in the parafoveal region and vitreous traction. 15 We found that older patients tended to have less average retinal thickness $(\mathrm{p}=0.15$, Table 5$)$, probably because vitreoretinal traction weakens with age.

It is noteworthy that patients who presented later also had larger apical areas (Table 4). Therefore, some TMHs may enlarge while the retina thickens over time. However, some TMHs spontaneously close, especially among young patients.10,15,25 Among 18 eyes of 18 consecutive patients (average age 14.9 years) with TMHs, eight (44\%) experienced spontaneous closure within 4 months of follow up.10 One possible mechanism involves glial and retinal pigment epithelial cell proliferation from the edges of the hole to fill the bottom of the hole, then through contractile forces pull the neurosensory retinal tissues together.26 Obviously hole closure would decrease the apical area, which appears to contradict the results of our study. However, our study was not a longitudinal evaluation of TMHs and only enrolled patients with full-thickness defects at the time of evaluation. Our patients were also significantly older. Therefore, patients who were less likely to experience spontaneous closure might be disproportionately represented in our study.

\section{Factors affecting visual acuity in TMH patients}

A study of 36 eyes (6 eyes had TMHs) revealed that macular hole size and retinal thickness were negatively correlated with visual acuity.27 In our study, we found no correlation between the apical and basal areas of the macular hole and visual acuity. However, average retinal thickness was positively correlated with visual acuity. These contradictory results may partly be due to the different types of macular holes studied. In addition, although thinner retina may imply less severe edema, it may also indicate more extensive retinal atrophy. Therefore, retinal thickness alone is probably a poor predictor of visual acuity.

Despite the various morphologies on OCT, different types of TMHs did not differ in terms of visual acuity. This may be because all subjects in the current study had full-thickness foveal involvement, likely resulting in nonfoveal fixation in a large number of patients. It would be difficult to discern subtle differences in Snellen visual acuity even if they existed.

Patients who presented later after injury tended to have better visual acuity, though the correlation was not statistically significant $(\mathrm{p}=0.11$, Table 3$)$. One explanation is that patients with less vision loss may delay seeking medical attention. It is also possible that visual 
acuity may improve with time due to structural changes within the holes, or that the patients simply have more time to adapt to nonfoveal fixation.

\section{Limitations of the study}

There are many limitations to this study that may impact the applicability of the results. The study is retrospective and cross-sectional, not longitudinal. Therefore, it cannot accurately describe the evolution of the morphometric and clinical parameters of traumatic macular holes. The clinical setting where the data are obtained was challenging, as many patients came from far away via an inadequate transportation system, making reliable follow up extremely difficult. Some patients may not be able to seek medical attention due to limited access to the health care system. Therefore, it was possible that a selection bias existed among patients who were included in the study. The low prevalence of TMHs, especially without other comorbidities, added another layer of difficulty to patient recruitment. Despite these difficulties, however, this is the largest published series of patients with traumatic macular holes to date.

The limitations of StratusOCT, which necessitated some assumptions in our data collection and analysis, are also evident. Centration of macular scans of fovea-involving pathology in patients with poor vision was difficult and often required multiple attempts. Therefore, prolonged scan time was impractical. Because the vertical and horizontal line scans had to be performed separately, there was no guarantee that they would intersect at the center of the hole. Since only two scans were performed on each patient, areas of the hole that lie outside of the scans were missed. Therefore, in cases where the hole was irregular (non-circular), some of the investigated parameters (area, eccentricity) had to be approximated. If the scanned areas were not representative, the retinal thickness measurements, and therefore the classification scheme, would also be inaccurate. However, despite these limitations, we believe this study provides some insight into the variable morphology of TMHs.

In summary, we presented the morphological and clinical characteristics of a consecutive series of full-thickness traumatic macular holes, which can be divided into five types based on their distinct appearance on OCT. In the future, spectral domain OCT, with a much faster data acquisition speed and sampling density, may facilitate a more comprehensive assessment of traumatic macular holes.

\section{Acknowledgments}

Supported in part by: Grant 2007A060305009 from Guangdong Science and Technology Institute, Grants EY 11753 and EY 03040 from the National Eye Institute and the National Center on Minority Health and Health Disparities, National Institutes of Health, Bethesda, MD, and an unrestricted grant from the Research to Prevent Blindness, New York, NY.

\section{References}

1. Gass, JDM. Stereoscopic atlas of macular diseases: diagnosis and treatment. 4th ed.. Mosby; St. Louis, MO: 1997. p. 904-937.

2. Altaweel M, Ip M. Macular hole: improved understanding of pathogenesis, staging, and management based on optical coherence tomography. Semin Ophthalmol. 2003; 18:58-66. [PubMed: 14566624] 
3. Hagemann LF, Costa RA, Ferreira HM, et al. Optical coherence tomography of a traumatic Neodymium:YAG laser-induced macular hole. Ophthalmic Surg Lasers Imaging. 2003; 34:57-59. [PubMed: 12570007]

4. Knapp H. Ueber Isolirte zerreissungen der aderhaut infolge von traumen auf augapfel. Arch Augenheilkd. 1869; 1:6-29.

5. Oglivie F. One of the results of concussion injuries of the eye. Trans Ophthalmol Soc UK. 1900; 20:202-229.

6. Yanagiya N, Akiba J, Takahashi M, et al. Clinical characteristics of traumatic macular hole. Jpn J Ophthalmol. 1996; 40:544-547. [PubMed: 9130060]

7. Mizusawa Y, Ichibe M, Yoshizawa T, et al. Clinical evaluation of traumatic macular hole. Jpn Rev Clin Ophthalmol. 1996; 90:790-792.

8. Kuhn F, Morris R, Mester V, et al. Internal limiting membrane removal for traumatic macular holes. Ophthalmic Surg Lasers. 2001; 32(4):308-315. [PubMed: 11475397]

9. Arevalo JF, Sanchez JG, Costa RA, et al. Optical coherence tomography characteristics of fullthickness traumatic macular holes. Eye. 2007 epub abstract.

10. Yamashita T, Uemara A, Uchino E, et al. Spontaneous closure of traumatic macular hole. Am J of Ophthalmol. 2002; 133:230-235. [PubMed: 11812427]

11. Coats G. The pathology of macular hole. Roy London Ophthalmic Hosp Rep. 1907; 17:69-96.

12. Delori F, Pomerantzeff O, Cox MS. Deformation of the globe under high-speed impact: its relation to contusion injuries. Invest Ophthalmol. 1969; 8:290-301. [PubMed: 5772720]

13. Johnson RN, McDonald, Lewis H, et al. Traumatic macular hole: observations, pathogenesis, and results of vitrectomy surgery. Ophthalmology. 2001; 108:853-857. [PubMed: 11320012]

14. Hirata A, Tanihara H. Ruptured internal limiting membrane associated with blunt trauma revealed by indocyanine green staining. Graefes Arch Clin Exp Ophthalmol. 2004; 242:527-530. [PubMed: 14986009]

15. Yamada H, Sakai A, Yamada E, et al. Spontaneous Closure of Traumatic Macular Hole. Am J Ophthalmol. 2002; 134:340-347. [PubMed: 12208244]

16. Yokozuka K, Kishi S, Tobe K, et al. Clinical features of traumatic macular hole. Jpn J Clin Ophthalmol. 1991; 45:1121-1124.

17. Liu, X. Clinical application of optical coherence tomography in ophthalmology. Guangdong Scientific Publishing Co; Guangzhou, China: 2006. p. 290In Chinese

18. Tornambe PE. Macular hole genesis: the hydration theory. Retina. 2003; 23:421-424. [PubMed: 12824853]

19. Oehrens AM, Stalmans P. Optical coherence tomographic documentation of the formation of a traumatic macular hole. Am J Ophthalmol. 2006; 142:866-869. [PubMed: 17056374]

20. Amari F, Ogino N, Matsumura M, et al. Vitreous surgery for traumatic macular holes. Retina. 1999; 19:410-413. [PubMed: 10546936]

21. De Bustros S. Vitreous surgery for traumatic macular hole. Retina. 1996; 16:451-452. [PubMed: 8912978]

22. Liu X, Ling Y, Gao R, et al. Optical coherence tomography's diagnostic value in evaluating surgical impact on idiopathic macular hole. Chin Med J. 2003; 116:444-447. [PubMed: 12781056]

23. Hee MR, Puliafito CA, Wong C, et al. Optical coherence tomography of macular holes. Ophthalmology. 1995; 102:748-756. [PubMed: 7777274]

24. Puliafito CA, Hee MR, Lin CP, et al. Imaging of macular diseases with optical coherence tomography. Ophthalmology. 1995; 102:217-229. [PubMed: 7862410]

25. Carpineto P, Ciancaglini M, Aharrh-Gnama A, et al. Optical coherence tomography and fundus microperimetry imaging of spontaneous closure of traumatic macular hole: A case report. Eur J Ophthalmol. 2005; 15:165-169. [PubMed: 15751261]

26. Menchini U, Virgili G, Giacomelli G, et al. Mechanism of spontaneous closure of traumatic macular hole: OCT study of one case. Retina. 2003; 23:104-106. [PubMed: 12652242]

27. Wei W, Yang W, Zhao L, et al. Optical coherence tomography of macular holes. Zhonghua Yan Ke Za Zhi. 1999; 35:419-421. In Chinese. [PubMed: 11835850] 


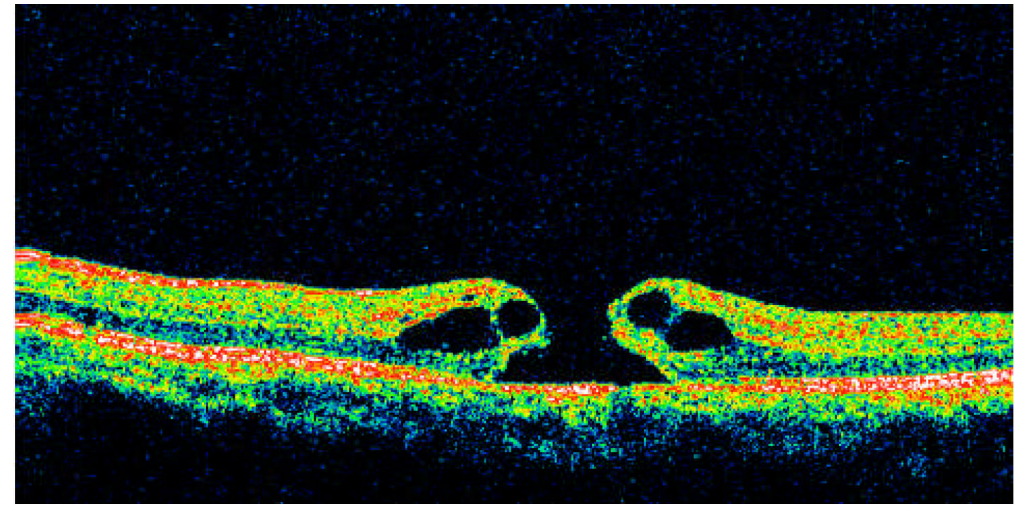

Fig. 1.

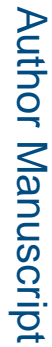




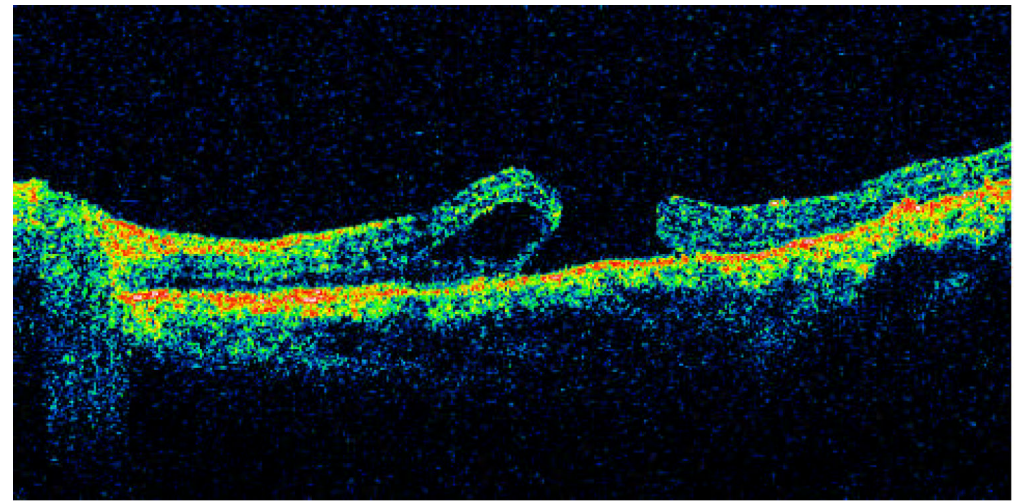

Fig. 2. 


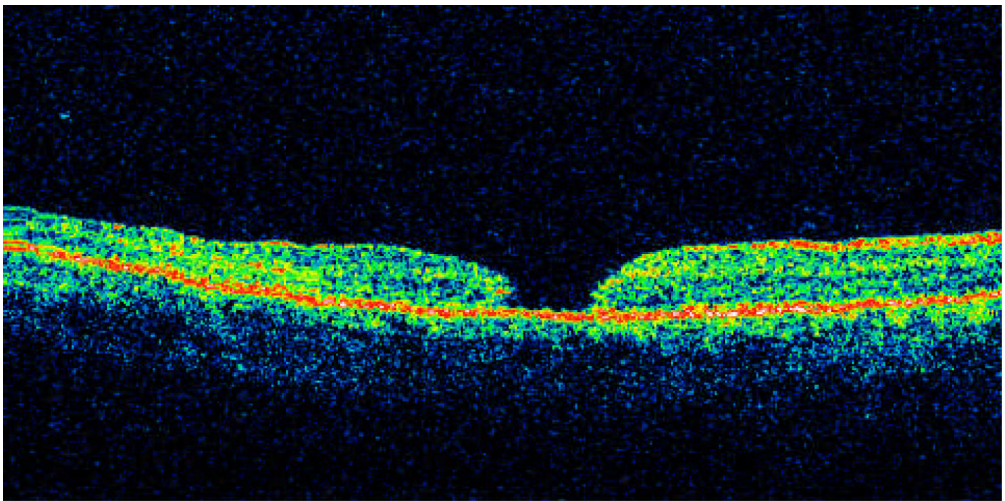

Fig. 3.

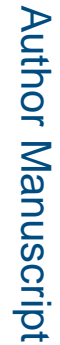




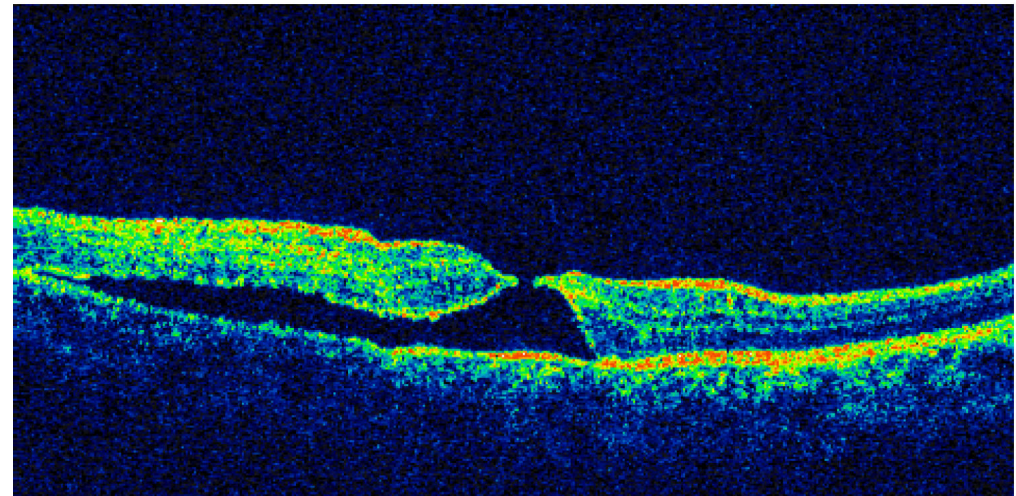

Fig. 4.

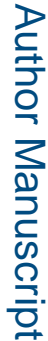




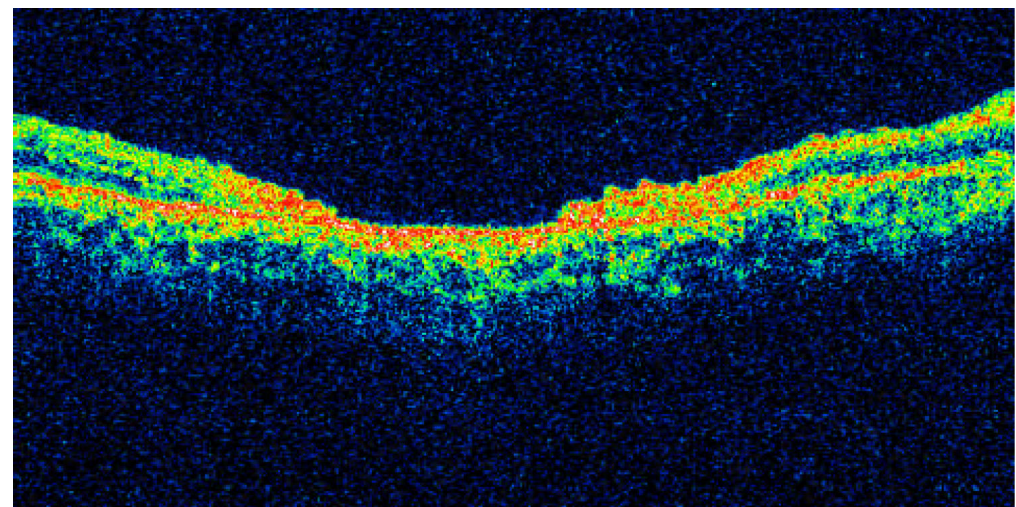

Fig. 5.

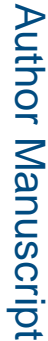




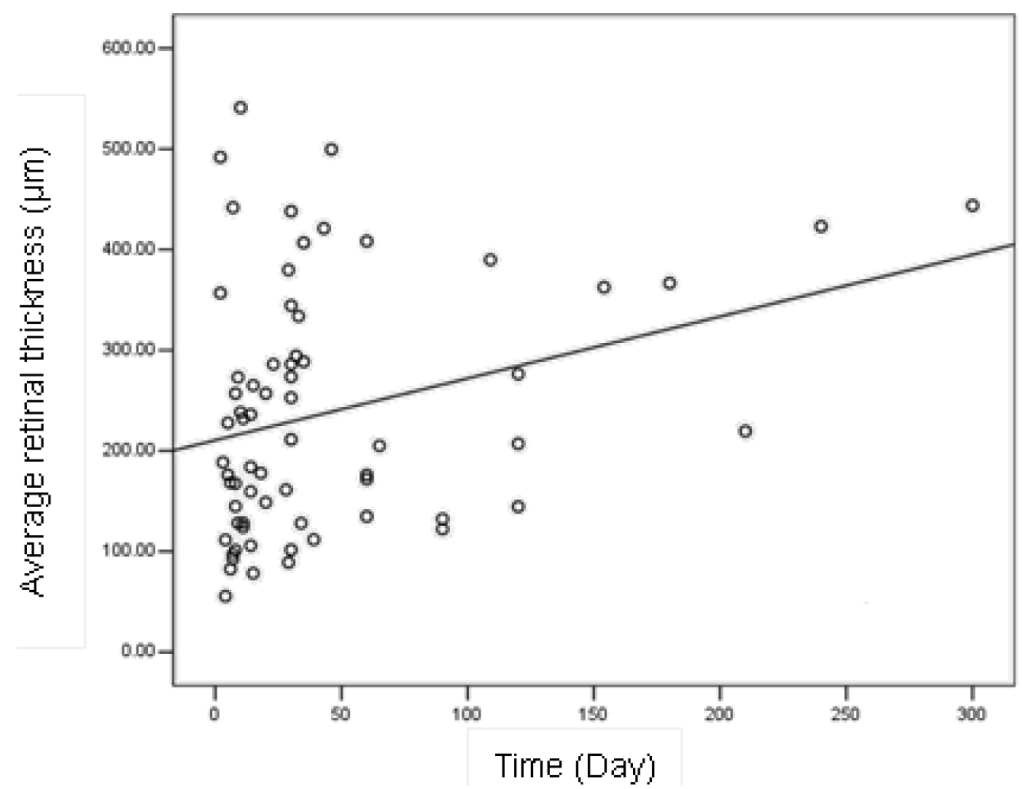

Figure 6. 


\section{롤 \\ }

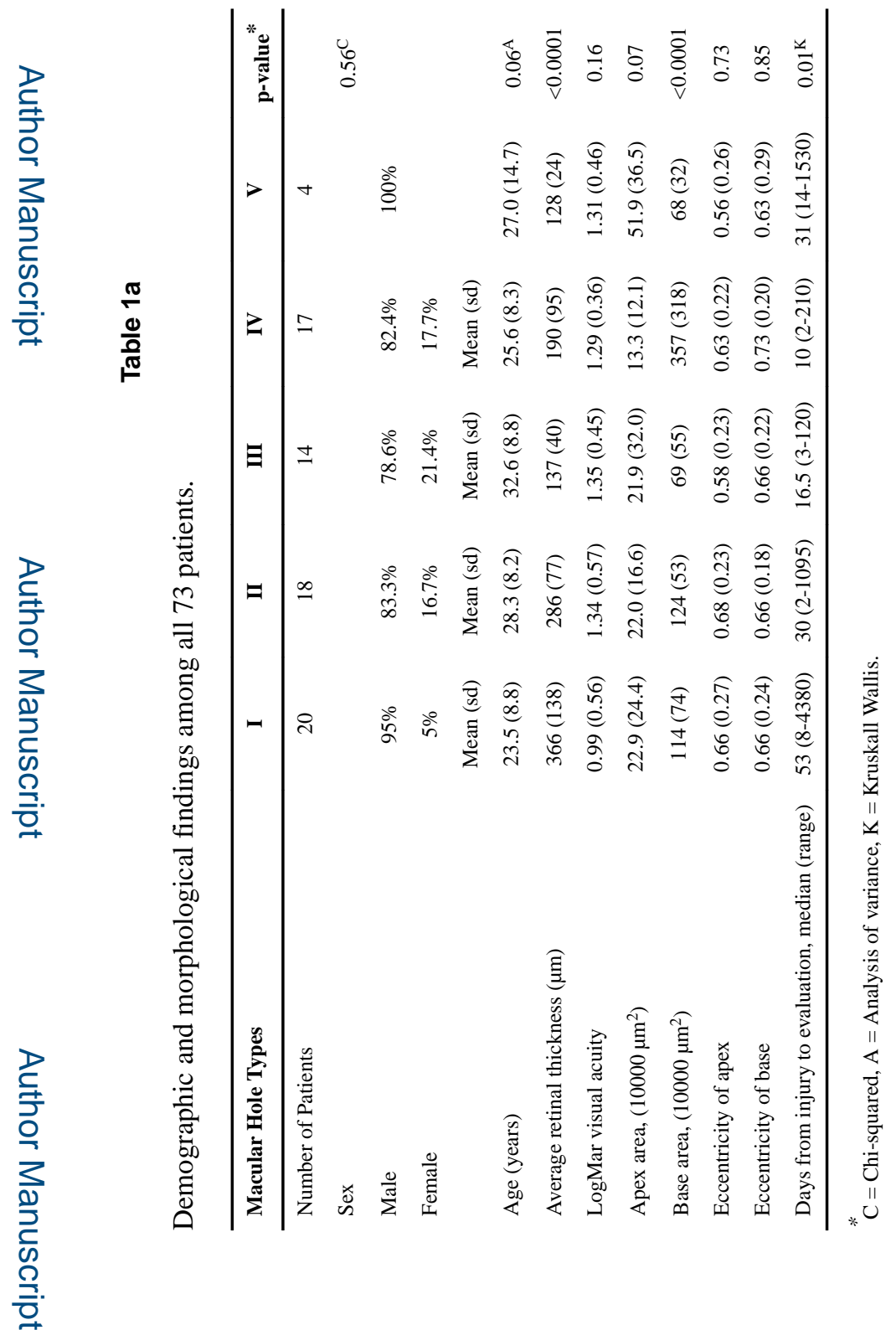

Retina. Author manuscript; available in PMC 2017 March 07. 


\section{을 \\ }

胥

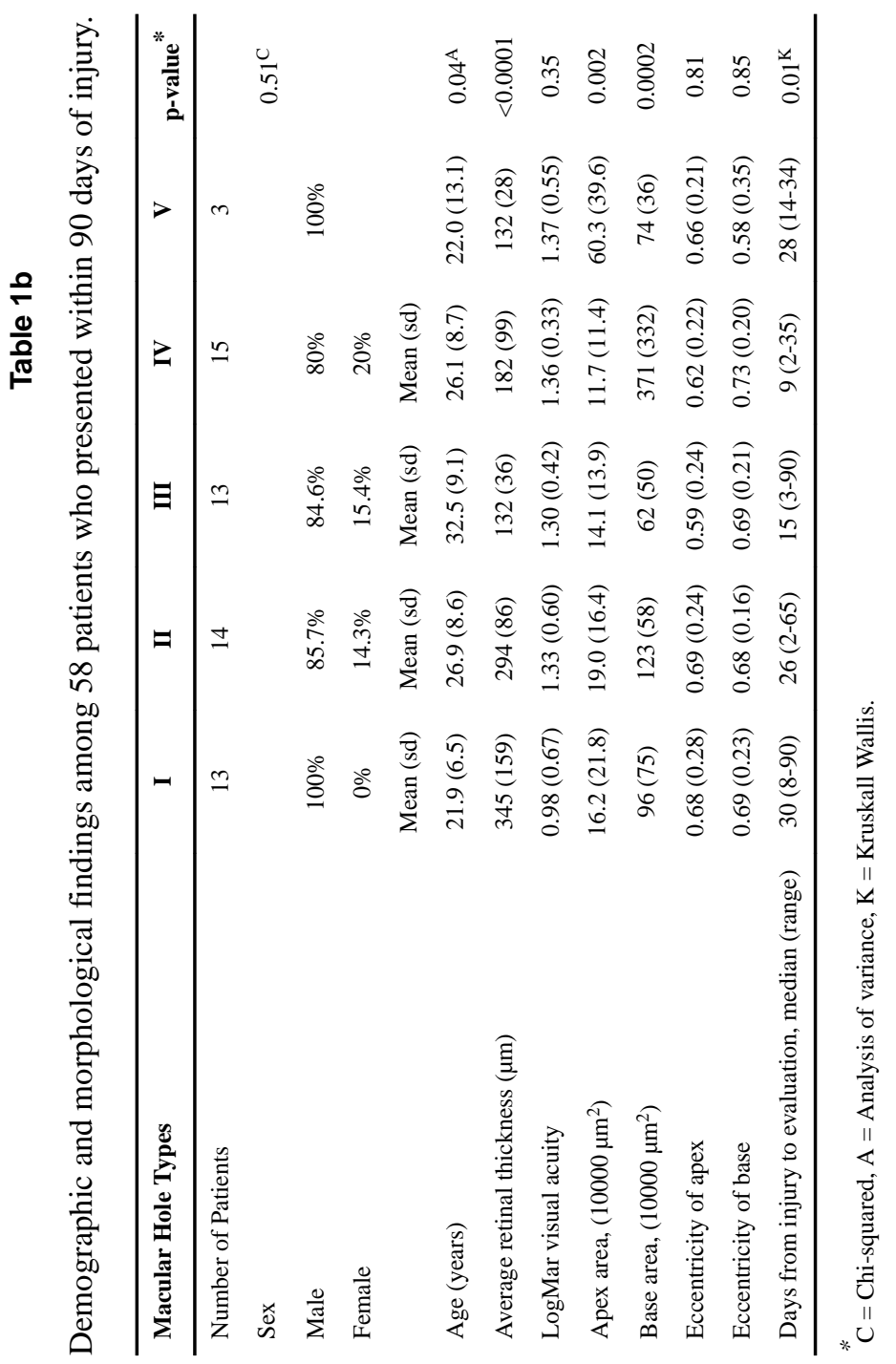

Retina. Author manuscript; available in PMC 2017 March 07. 


\section{Table 2}

Relationship between type of macular hole and the time from injury to evaluation.

\begin{tabular}{|c|c|c|c|}
\hline & $\begin{array}{l}\text { Patients who presented within days of injury, } \\
\text { n (row \%) }\end{array}$ & $\begin{array}{c}\text { Patients who } 90 \text { presented }<90 \text { day from injury, } \\
\text { n (row \%) }\end{array}$ & Chi-square p-value \\
\hline Macular hole & & & 0.29 \\
\hline Type I & $13(65.0 \%)$ & $7(35.0 \%)$ & \\
\hline Type II & $14(77.8 \%)$ & $4(22.2 \%)$ & \\
\hline Type III & $13(92.9 \%)$ & $1(7.1 \%)$ & \\
\hline Type IV & $15(88.2 \%)$ & $2(11.8 \%)$ & \\
\hline Type V & $3(75.0 \%)$ & $1(25.0 \%)$ & \\
\hline
\end{tabular}




\section{Table 3}

Correlations with logMar visual acuity.

\begin{tabular}{lc}
\hline & LogMar visual acuity Pearson Correlation Coefficient \\
\hline Area (apex) & $0.013 \mathrm{p}=0.92 \mathrm{n}=71$ \\
Area (base) & $-0.0014 \mathrm{p}=0.99 \mathrm{n}=70$ \\
Area (apex) & $-0.13 \mathrm{p}=0.58 \mathrm{n}=20$ \\
Type I & $0.05 \mathrm{p}=0.85 \mathrm{n}=17$ \\
Type II & $0.40 \mathrm{p}=0.15 \mathrm{n}=14$ \\
Type III & $-0.11 \mathrm{p}=0.69 \mathrm{n}=16$ \\
Type IV & $-0.48 \mathrm{p}=0.52 \mathrm{n}=4$ \\
Type V & \\
Area (base) & $-0.17 \mathrm{p}=0.47 \mathrm{n}=20$ \\
Type I & $-0.35 \mathrm{p}=0.17 \mathrm{n}=17$ \\
Type II & $0.40 \mathrm{p}=0.10 \mathrm{n}=14$ \\
Type III & $-0.01 \mathrm{p}=0.96 \mathrm{n}=15$ \\
Type IV & $-0.26 \mathrm{p}=0.74 \mathrm{n}=4$ \\
Type V & $-0.10 \mathrm{p}=0.40$ \\
Eccentricity (apex) & $-0.017 \mathrm{p}=0.89$ \\
Eccentricity (base) & $-0.23 \mathrm{p}=0.07$ \\
Average retinal thickness & \\
\hline & LogMar visual acuity Spearman Correlation Coefficient \\
\hline & $-0.19 \mathrm{p}=0.11$ \\
\hline Time from injury to exam &
\end{tabular}




\section{Table 4}

Differences between patients who presented early vs. late after injury.

\begin{tabular}{lccc}
\hline & $\begin{array}{c}\text { Patients who presented within 90 days of } \\
\text { injury }(\mathbf{n = 5 8}) \text { mean }(\mathbf{s d})\end{array}$ & $\begin{array}{c}\text { Patients who present }>90 \text { days after } \\
\text { injury }(\mathbf{n = 1 5}) \text { mean }(\mathbf{s d})\end{array}$ & \multicolumn{2}{c}{ T-test p-value } \\
\hline Average retinal thickness $(\mu \mathrm{m})$ & $232(131)$ & $313(111)$ & 0.03 \\
Apex area $\left(10000 \mu \mathrm{m}^{2}\right)$ & $17.5(20.0)$ & $38.4(30.1)$ & 0.002 \\
Base area $\left(10000 \mu \mathrm{m}^{2}\right)$ & $161(208)$ & $150(93)$ & 0.84 \\
Eccentricity of apex & $0.645(0.240)$ & $0.614(0.234)$ & 0.65 \\
Eccentricity of base & $0.690(0.204)$ & $0.624(0.246)$ & 0.29 \\
\hline
\end{tabular}


Table 5

Correlations with age.

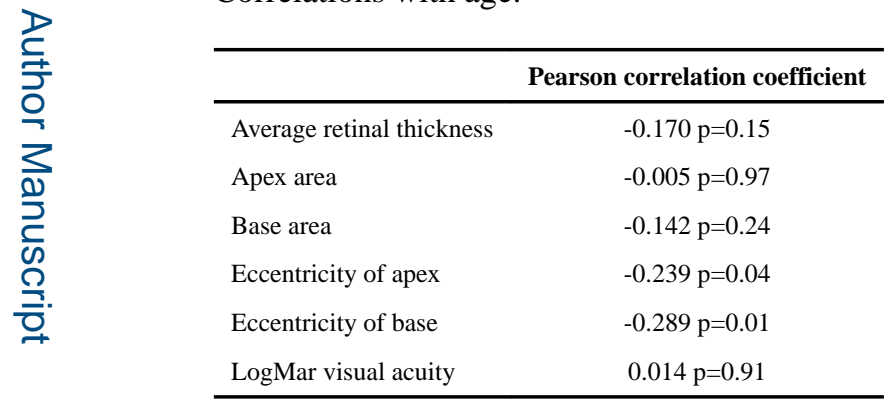

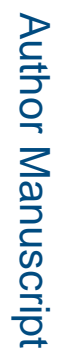

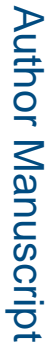

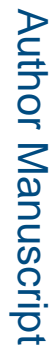

Retina. Author manuscript; available in PMC 2017 March 07. 